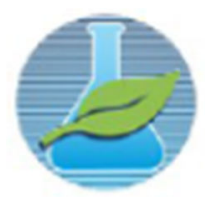

Vol. 02 N. 04 (2016) 040-042

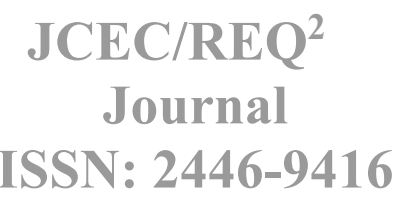

"EU, A INDÚSTRIA E O MUNDO"

08 a 11 de novembro de 2016 no campus Viçosa da UFV

Departamento de Engenharia de Produção e Mecânica - DEP

Universidade Federal de Viçosa - UFV

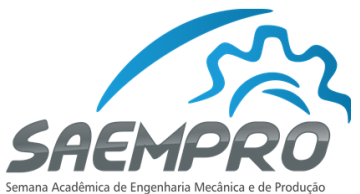

doi: https://doi.org/10.18540/2446941602042016040

OPEN ACCESS

\title{
MODELAGEM E SIMULAÇÃO CFD DE TROCADOR DE CALOR PARA CONDICIONAMENTO DE AR
}

\author{
Luísa Fernanda Martins Ribeiro Giardini, Álvaro Messias Binhonha Tibiriça \\ Universidade Federal de Viçosa, Departamento de Engenharia de Produção e Mecânica \\ Ph. Rolfs s/n - 36570-000 - Viçosa - MG \\ luisa.giardini@ufv.br, alvaro.tibirica@ufv.br
}

\section{INTRODUÇÃO}

Em trocadores de calor, o lado do ar representa uma resistência de troca de calor de $85 \%$. Como resultado, para melhorar o desempenho térmico e reduzir o tamanho e o peso, são acrescentadas aletas (superfícies, normalmente metálicas, com alto coeficiente de condução) para aumentar a superfície de contato (Bhuiyan et al., 2012). Principal componente em sistemas de ar condicionado, trocadores de calor do tipo tubo aletado são representantes de trocadores de calor compacto devido a sua elevada capacidade de troca de calor (Dogan et al., 2015). A transferência de calor ocorre por meio de um escoamento cruzado sobre bancos de tubos. Em tais equipamentos, um fluido moverá dentro dos tubos enquanto o outro (ar no caso de um sistema de ar condicionado) se move perpendicularmente aos tubos, direcionado por aletas (Çengel et al., 2012).

A construção civil responde por um terço proporção de todo o consumo de energia na sociedade. Mais da metade desse consumo de energia provém de aquecimento, ventilação e sistemas de ar condicionado. Dessa forma, com o intuito de aumentar a eficiência desses sistemas e consequentemente dos trocadores de calor (responsáveis pela transferência de calor), vários estudos são feitos com o intuito de melhorar o desempenho dos trocadores de calor (Dogan et al., 2015; Jiang et al., 2015). O fluxo de ar através de um trocador de calor compacto é complexo. Por isso a previsão teórica de coeficientes de transferência de calor não é facilmente determinada (Wang et al., 1996). Por fornecer flexibilidade da utilização e de uma ampla variedade de condições físicas através de modelos computacionais realistas sem que haja necessidade de construir equipamentos de teste caros ou protótipos, a simulação CFD (Computacional Fluid Dynamics) tem contribuído com esses estudos (Yaïci et al., 2014).

\section{OBJETIVO}

Validar um modelo computacional com base nos dados experimentais obtidos por Wang et al. (1996).

\section{METODOLOGIA}

O trabalho desenvolvido por Wang et al. (1996) testou 15 trocadores de calor com diferentes dimensões entre si e para diferentes valores de velocidade. Principais parâmetros para análise em um trocador de calor, transferência de calor e queda de pressão foram obtidas e adimensionadas através do fator $\mathrm{j}$ de Colburn e do fator $\mathrm{f}$ de atrito, respectivamente. No fim, resultou em correlações dependentes do número de Reynolds $\left(\operatorname{Re}_{\mathrm{D}}\right)$ e de parâmetros da geometria do trocador de calor $(t=$ espessura da aleta; $D=$ diâmetro do tudo; $N=$ número de tubos; $F_{p}=$ distância entre aletas) -Eq. (1) e (2). 


$$
\begin{aligned}
& j=0,394 R e_{D}-0,392\left(\frac{t}{D}\right)^{-0,0449} N^{-0,0897}\left(\frac{F p}{D}\right)^{-0,212} \\
& f=1,039 R e_{D}^{-0,418}\left(\frac{t}{D}\right)^{-0,104} N^{-0,0935}\left(\frac{F p}{D}\right)^{-0,197}
\end{aligned}
$$

Escolheu-se um trocador dentre os 15 testados por Wang et al. (1996) e o modelo computacional por modelagem CFD foi criado a partir das dimensões do mesmo $(N=4$ tubos; $F p=$ $3 \mathrm{~mm} ; D=10,23 \mathrm{~mm} ; t=0,13 \mathrm{~mm} ; P t$ (distância transversal entre tubos) $=25,4 \mathrm{~mm} ; P l$ (distância longitudinal entre tubos $)=22 \mathrm{~mm} ; L$ (tamanho total do trocador $)=88 \mathrm{~mm})$. Aproveitando o fato do trocador de calor possuir simetrias, um modelo 3D simplificado foi criado (Fig. 1).
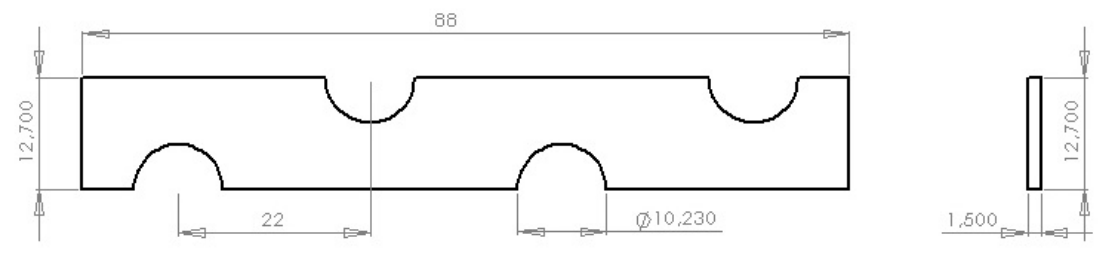

Figura 1 - Geometria criada e respectivas dimensões.

O modelo 3D foi divido em elementos finitos (malha). Tomou-se o cuidado de obter elementos menores próximos a paredes para que assim os fenômenos de camadas limites fossem capturados. Além disso, um teste de malha foi feito a fim de escolher a que gerasse melhores resultados com a menor exigência computacional.

As condições de contorno foram definidas de acordo com as velocidades testas por Wang et al. (1996) e as demais para que se igualasse ao modelo real. Inlet para a entrada, wall para tubos e aleta, symmetry para laterais e no plano médio entre duas aletas e openning para a saída do ar. E o modelo $\mathrm{k}-\omega$ de turbulência foi adotado, pois é o que consegue captar os fenômenos das camadas limites em conjunto com a malha criada.

As equações utilizadas para comparação às correlações de Wang et al. (1996) estão descritas abaixo - Eq. (3), (4) e (5). O comprimento característico utilizado por Wang et al. (1996) foi o diâmetro do tubo $(D)$, porém nesse presente trabalho, ele foi substituído pela distância entre as aletas $(F p)$. Onde utilizou-se o adimensional Pranlt $(P r)$, a condutividade do ar $(k)$, o fluxo de calor $(\dot{Q})$, temperatura $(T)$, pressão $(P)$, densidade do $\operatorname{ar}(\rho)$ e velocidade $(v)$.

$$
\begin{aligned}
& j=\frac{h F_{P}}{k R e_{F p} P r^{\frac{1}{3}}} \\
& h=\frac{\dot{Q}}{T_{\text {parede }}-T_{\text {entrada }}} \\
& f=\frac{P_{\text {entrada }}-P_{\text {saida }}}{\frac{1}{2} \rho v^{2} 4 L}
\end{aligned}
$$

\section{RESULTADOS E DISCUSSÃO}

Os resultados encontrados estão dispostos na Fig. 2 e Fig. 3. Representam as curvas dos dados obtidos experimentalmente (Wang et al., 1996) e os dados obtidos pelo modelo computacional gerado por esse trabalho para cinco valores de Reynolds. 


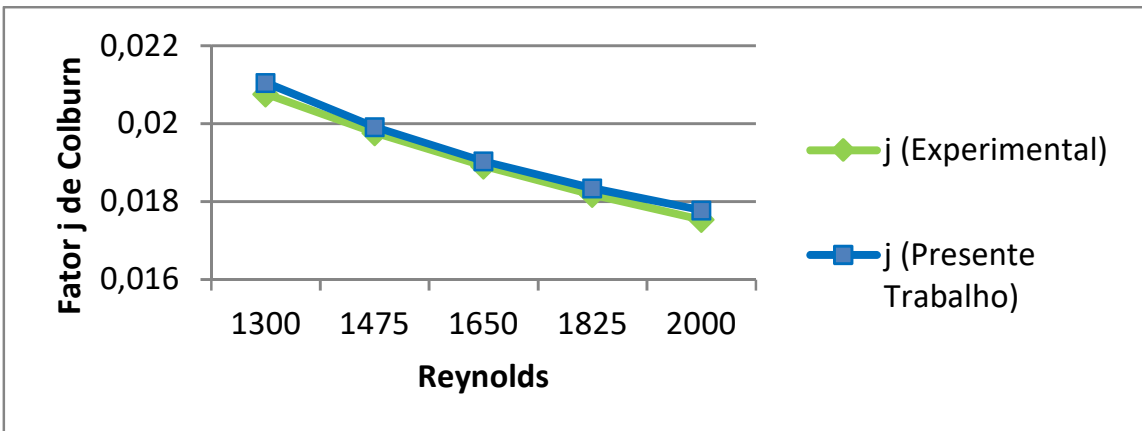

Figura 2 - Fator j de Colburn em função do número de Reynolds dos dados experimentais e do modelo computacional obtido.

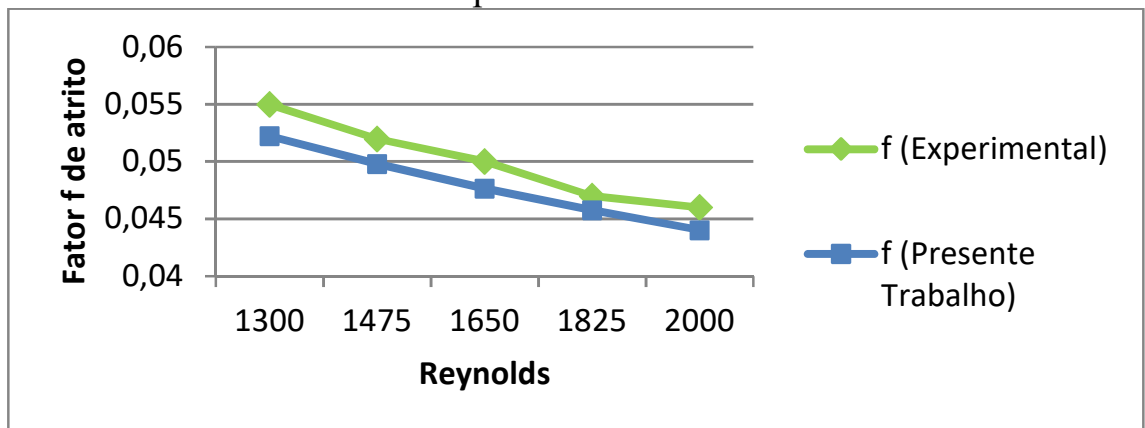

Figura 3 - Fator f de atrito em função do número de Reynolds dos dados experimentais e do modelo computacional obtido.

$\mathrm{O}$ fator $\mathrm{j}$ de Colburn e o fator $\mathrm{f}$ de atrito decrescem com o aumento do número de Reynolds, mesmo a transferência de calor e a queda de pressão aumentando. Isso se deve ao fato dos adimensionais serem inversamente proporcionais à velocidade. Os valores do fator $\mathrm{j}$ de Colburn obtido pelo modelo computacional aproximaram dos dados experimentais em uma faixa menor que $1,4 \%$ e os dados do fator $\mathrm{f}$ de atrito do modelo computacional aproximaram dos dados experimentais em uma faixa menor que $5,05 \%$.

\section{CONCLUSÃO}

O presente trabalho aproximou os resultados em valores inferiores a 5,05\% dos resultados experimentais obtidos por Wang et al. (1996). Dessa forma, conclui-se que o modelo computacional obtido pode ser utilizado para representar a realidade e com isso para futuras pesquisas.

\section{REFERÊNCIAS}

BHUIYAN, A. A.; AMIN, M, R.; ISLAM, A.K.M.S. "Three-dimensional performance analysis of plain fin tube heat exchangers in transitional regime". Applied Thermal Engineering. p. 445-454, 2013.

ÇENGEL, Y.A; GHAJAR, A.J. "Transferência de Calor e massa: uma abordagem prática". Porto Alegre: AMGH, 4 ed., 2012.

DOGAN, B; ALTUN, O; UGURLUBILEK, N; TOSUN, M; SARIÇAY, T.; ERBAY, L.B. “An experimental comparison of two multi-louvered fin heat exchangers with different numbers of fin rows". Applied Thermal Engineering. p. 270-278, 2015.

WANG, C.; CHANG, Y.; HSIEH Y.; LIN, Y. "Sensible heat and friction characteristics of plate finand-tube heat exchangers having plane fins". International Journal of Refrigeration 19, p.223230, 1996.

YAÏCI, W; GHORAB, M; GHORAB, E.; " $3 D$ CFD analysis of the effect of inlet air flow maldistribution on the fluid flow and heat transfer performances of plate-fin-and-tube laminar heat exchangers". International Journal of Heat and Mass Transfer 74, p. 490-500, 2014. 